\title{
Tracking and Improving Information in the Service of Fairness
}

\author{
SUMEGHA GARG ${ }^{*}$, Princeton University \\ MICHAEL P. KIM ${ }^{\dagger}$ and OMER REINGOLD ${ }^{\ddagger}$, Stanford University
}

\begin{abstract}
As algorithmic prediction systems have become widespread, fears that these systems may inadvertently discriminate against members of underrepresented populations have grown. With the goal of understanding fundamental principles that underpin the growing number of approaches to mitigating algorithmic discrimination, we investigate the role of information in fair prediction. A common strategy for decision-making uses a predictor to assign individuals a risk score; then, individuals are selected or rejected on the basis of this score. In this work, we study a formal framework for measuring the information content of predictors. Central to the framework is the notion of a refinement; intuitively, a refinement of a predictor $z$ increases the overall informativeness of the predictions without losing the information already contained in $z$. We show that increasing information content through refinements improves the downstream selection rules across a wide range of fairness measures (e.g. true positive rates, false positive rates, selection rates). In turn, refinements provide a simple but effective tool for reducing disparity in treatment and impact without sacrificing the utility of the predictions. Our results suggest that in many applications, the perceived "cost of fairness" results from an information disparity across populations, and thus, may be avoided with improved information.
\end{abstract}

CCS Concepts: • Theory of computation $\rightarrow$ Theory and algorithms for application domains; $\bullet$ Mathematics of computing $\rightarrow$ Information theory.

Additional Key Words and Phrases: Algorithmic fairness, prediction

\section{ACM Reference Format:}

Sumegha Garg, Michael P. Kim, and Omer Reingold. 2019. Tracking and Improving Information in the Service of Fairness. In ACM EC '19: ACM Conference on Economics and Computation (EC '19), June 24-28, 2019, Phoenix, AZ, USA. ACM, New York, NY, USA, 16 pages. https://doi.org/10.1145/3328526.3329624

\section{INTRODUCTION}

As algorithmic predictions are increasingly employed as parts of systems that classify people, concerns that such classfiers may be biased or discriminatory have increased correspondingly. These concerns are far from hypothetical; disparate treatment on the basis of sensitive features, like race and gender, has been well-documented in diverse algorithmic application domains $[3,5,12]$. As such, researchers across fields like computer science, machine learning, and economics have responded with many works aiming to address the serious issues of fairness and unfairness that arise in automated decision-making systems.

\footnotetext{
*Part of this work completed while visiting Stanford University.

${ }^{\dagger}$ Part of this work completed while visiting the Weizmann Institute of Science. Supported, in part, by a Google Faculty Research Award, CISPA Center for Information Security, and the Stanford Data Science Initiative.

† Supported by NSF Grant CCF-1763311.
}

Permission to make digital or hard copies of all or part of this work for personal or classroom use is granted without fee provided that copies are not made or distributed for profit or commercial advantage and that copies bear this notice and the full citation on the first page. Copyrights for components of this work owned by others than the author(s) must be honored. Abstracting with credit is permitted. To copy otherwise, or republish, to post on servers or to redistribute to lists, requires prior specific permission and/or a fee. Request permissions from permissions@acm.org.

EC '19, fune 24-28, 2019, Phoenix, AZ, USA

(c) 2019 Copyright held by the owner/author(s). Publication rights licensed to ACM.

ACM ISBN 978-1-4503-6792-9/19/06 ..\$15.00

https://doi.org/10.1145/3328526.3329624 
While most researchers studying algorithmic fairness can agree on the high-level objectives of the field (e.g., to ensure individuals are not mistreated on the basis of protected attributes; to promote social well-being and justice across populations), there is much debate about how to translate these normative aspirations into a concrete, formal definition of what it means for a prediction system to be fair. Indeed, as this nascent field has progressed, the efforts to promote "fair prediction" have grown increasingly divided, rather than coordinated. Exacerbating the problem, [28] identifies that each new approach to fairness makes its own set of assumptions, often implicitly, leading to contradictory notions about the right way to approach fairness [7, 26]; these inconsistencies add to the "serious challenge for cataloguing and comparing defintions" [28]. Complicating matters further, recent works $[8,27]$ have identified shortcomings of many well-established notions of fairness. At the extreme, these works argue that blindly requiring certain statistical fairness conditions may in fact harm the communities they are meant to protect.

The state of the literature makes clear that choosing an appropriate notion of fairness for prediction tasks is a challenging affair. Increasingly, fairness is viewed as a context-dependent notion [19, 31], where the "right" notion for a given task should be informed by conversations between computational and social scientists. In the hopes of unifying some of the many directions of research in the area, we take a step back and ask whether there are guiding princples that broadly serve the high-level goals of "fair" prediction, without relying too strongly on any specific notion of fairness. The present work argues that understanding the "informativeness" of predictions needs to be part of any sociotechnical conversation about the fairness of a prediction system. Our main contribution is to provide a technical language with strong theoretical backing to discuss informational issues in the context of fair prediction.

Our contributions. Towards the goal of understanding common themes across algorithmic fairness, we investigate the role of information in fair prediction. We study a formal notion of informativeness in predictions and demonstrate that it serves as an effective tool for understanding and improving the utility, fairness, and impact of downstream decisions. In short, we identify that many "failures" of requiring fairness in prediction systems can be explained by an information disparity across subpopulations. Importantly, the framework is not wedded to a specific fairness desideratum and can be applied broadly to prediction settings where discrimination may be a concern. Our main contributions can be summarized as follows:

- First and foremost, we identify informativeness as a key fairness desideratum. We provide information-theoretic and algorithmic tools for reasoning about how much an individual's prediction reveals about their eventual outcome. Our formulation clarifies the intuition that more informative predictions should enable fairer outcomes, across a wide array of interpretations of what it means to be "fair." Notably, calibration plays a key technical role in this reasoning; the information-theoretic framework we present relies intimately on the assumption that the underlying predictors are calibrated. Indeed, our results demonstrate a surprising application of these calibration-based methods towards improving parity-based fairness criteria, running counter to the conventional wisdom that calibration and parity are completely at odds with one another.

- In Section 3, we provide a self-contained exposition of the framework we use to study the information content of a predictor. Information content formally quantifies the uncertainty over individuals' outcomes given their predictions. Leveraging properties of calibrated predictors, we show that the information content of a predictor is directly related to the information loss between the true risk distribution and the predicted risk distribution. Therefore, in many cases, information content - a measurable characteristic of the predicted risk distribution can serve as a proxy for reasoning about the information disparity across groups. To compare 
the information content of multiple predictors, we need a key concept called a refinement; informally, a refinement of a predictor increases the overall information content, without losing any of the original information. Refinements provide the technical tool for reasoning about how to improve prediction quality.

- In Section 4, we revisit the question of finding an optimal fair selection rule. For prominent parity-based fairness desiderata, we show that the optimal selection rule can be characterized as the solution to a certain linear program, based on the given predictor. We prove that improving the information content of these predictions via refinements results in a Pareto improvement of the resulting program in terms of utility, disparity, and long-term impact. As one concrete example, if we hold the selection rule's utility constant, then refining the underlying predictions causes the disparity between groups to decrease. Additionally, we prove that at times, the cost associated with requiring fairness should be blamed on a lack of information about important subpopulations, not on the fairness desideratum itself.

- In Section 5, we describe a simple algorithm, merge, for incorporating disparate sources of information into a single calibrated predictor. The merge operation can be implemented efficiently, both in terms of time and sample complexity. Along the way in our analysis, we introduce the concept of refinement distance - a measure of how much two predictors "overlap" - that may be of independent interest.

Finally, a high-level contribution of the present work is to clarify challenges in achieving fairness in prediction tasks. Our framework for tracking and improving information is particularly compelling because it does not requires significant technical background in information theory nor algorithms to understand. We hope the framework will facilitate interactions between computational and social scientists to further unify the literature on fair prediction, and ultimately, effect change in the fairness of real-world prediction systems.

\subsection{Why information?}

We motivate the study of information content in fair prediction by giving an intuitive overview of how information disparities can lead to unfair treatment and impact. We scaffold our discussion around examples from two recent works $[8,27]$ that raise concerns about using broad-strokes statistical tests as the definition of fairness. This overview will be informal, prioritizing intuition over technicality; see Section 2 for the formal preliminaries.

We consider a standard prediction setting where a decision maker, who we call the lender, has access to a predictor $z: \mathcal{X} \rightarrow[0,1]$; from the predicted risk score $z(x)$, the decison maker must choose whether to accept or reject the individual $x \in \mathcal{X}$, i.e. whether to give $x$ a loan or not. For each individual $x \in \mathcal{X}$, we assume there is an associated outcome $y \in\{0,1\}$ representing if they would default or repay a given loan ( 0 and 1 , respectively). Throughout, we will be focused on calibrated predictors. Intuitively, calibration requires that a predicted score of $z(x)=p$ corresponds to the same level of risk, regardless of whether $x \in A$ or $x \in B$. More technically, this means that we can think of $z(x)=p$ as a conditional probability; that is, amongst the individuals who receive score $z(x)=p$, a $p$-fraction of them end up having $y=1$. For simplicity, we assume there are two disjoint subpopulations $A, B \subseteq \mathcal{X}$. The works of $[8,27]$ mainly focus on settings where there are material differences between the distribution of $y$ in the populations $A$ and $B$, arguing that these differences can lead to undesirable outcomes. We argue that even if the true risk of individuals from $A$ and $B$ are identically distributed, differences in the distribution of predicted risk scores give rise to the same pitfalls.

A caution against parity. [27] focuses on notions of fairness that require parity between groups. One notion they study is demographic parity, which requires that the selection rate between 
groups $A$ and $B$ be equal; that is, $\operatorname{Pr}[x$ selected $\mid x \in A]=\operatorname{Pr}[x$ selected $\mid x \in B]$. Suppose that the majority of applicants come from group $A$ and that on-average, members of $A$ tend to have higher predictions according to $z$. In such a setting, an unconstrained utility-maximizing lender would give out loans at a higher rate in $A$ than in $B$. The argument in [27] against requiring demographic parity goes as follows: a lender who is constrained to satisfy demographic parity must either give out fewer loans in $A$ or more in $B$; because the lender does not want to give up utility from loaning to $A$, the constrained lender will give out more loans in $B$. [27] argue that in many reasonable settings, the lender will end up loaning to underqualified individuals in $B$ who are unlikely to repay; thus, the default rate in $B$ will increase significantly. In their model, this increased default rate translates into negative impact on the population $B$, whose members may go into debt and become even less creditworthy.

A caution against calibration. In general, [8] advocates for the use of calibrated score functions paired with threshold selection policies, where an individual is selected if $z(x)>\tau$ for some fixed, group-independent threshold $\tau$. Still, they caution that threshold policies paired with calibrated predictors are not sufficient to guarantee equitable treatment. In particular, suppose that the lender is willing to accept individuals if they have at least 0.7 probability of returning the loan. But now consider a set of calibrated risk scores where the scores are much more confident about $A$ than about $B$; at the extreme, suppose that for $x \in A, z(x) \in\{0,1\}$ (i.e. perfect predictions) and for $x \in B$, $z(x)=0.5$ (i.e. uniform predictions). In this case, using a fixed threshold of $\tau=0.7$ will select every qualified individual in $A$ and none of the individuals from $B$, even though, by the fact that $z$ is calibrated, half of them were qualified. Worse yet, even if we try to select more members of $B$, every member of $B$ has a 0.5 probability of defaulting. Indeed, in this example, we cannot distinguish between the individuals in $B$ because they all receive the same score $z(x)$. In other words, we have no information within the population $B$ even though the predictor was calibrated.

These examples make clear that when there are actual differences in the risk score distributions between populations $A$ and $B$, seemingly-natural approaches to ensuring fairness - enforcing parity amongst groups or setting a group-independent threshold - may result in a disservice to the underrepresented population. These works echo a perspective raised by [11] that emphasizes the distinction between requiring broad-strokes demographic parity as a constraint versus stating parity as a desideratum. Even if we believe that groups should ideally be treated similarly, defining fairness as satisfying a set of hard constraints may have unintended consequences.

Note that the arguments above relied on differences in the predicted risk scores $z(x)$ for $x \in A$ and $x \in B$, but not the true underlying risk. This observation has two immediate corollaries. On the one hand, in both of these vignettes, if the predicted score distributions are different between population $A$ and $B$, then such approaches to fairness could still cause harm, even if the true score distributions are identically distributed. On the other hand, just because $A$ and $B$ look different according to the predicted scores, they may not actually be different. Intuitively, the difference between the true risk distribution and the observed risk distribution represents a certain "information loss." Optimistically, if we could improve the predicted scores to reflect the underlying populations more accurately, then the resulting selection rule might exhibit less disparity between $A$ and $B$ in both treatment and impact.

Concretely, suppose we're given a set of predicted risk scores where the scores in $A$ tend to be much more extreme (towards 0 and 1 ) than those of $B$. Differences in the risk score distributions such as these can arise for one of two reasons: either individuals from $B$ are inherently more stochastic and unpredictable than those in $A$; or somewhere along the risk estimation pipeline, more information was lost about $B$ than about $A$. Understanding which story is true can be challenging, if not impossible. Still, in cases where we can reject the hypothesis that certain individuals are inherently 
less predictable than others, the fundamental question to ask is how to recover the lost information in our predictions. In this work, we provide tools to answer this question.

Refinements. Refinements provide the key tool for comparing the information of predictors. Intuitively, a calibrated predictor $\rho: \mathcal{X} \rightarrow[0,1]$ is a refinement of $z$ if $\rho$ hasn't forgotten any of the information contained by $z$. Formally, we say that $\rho$ refines $z$ if $\mathbf{E}_{x \sim \chi}[\rho(x) \mid z(x)=v]=v$; this definition is closely related to the idea of calibration in a sense that we make formal in Proposition 3.2. Refinements allow us to reason about how information influences a broad range of quantities of interest in the context of fair prediction. To give a sense of this, consider the following lemma, which we use to prove our main result in Section 4, but is also independently interesting.

LemMA. If $\rho$ is a refinement of $z$, then for all selection rates $\beta \in[0,1]$,

$$
\operatorname{TPR}^{\rho}(\beta) \geq \operatorname{TPR}^{z}(\beta), \quad \operatorname{FPR}^{\rho}(\beta) \leq \operatorname{FPR}^{z}(\beta), \quad \operatorname{PPV}^{\rho}(\beta) \geq \operatorname{PPV}^{z}(\beta) .
$$

Intuitively, the lemma shows that by improving information through refinements, mutliple key fairness quantities improve simultaneously. Leveraging this lemma and other properties of refinements and calibration, we show that for many different ways a decision-maker might choose their "optimal" selection rule, the "quality" of the selection rule improves under refinements.

Perspective. Disparities in the information content of risk scores may arise for many reasons. The present work clarifies how disparities across groups at early stages of the decision-making pipeline may contribute to disparities in the downstream decisions. In particular, differences in the availability or quality of training data as well as optimization procedures that are tailored for performance in the majority population could contribute to information loss in the minority. The present work highlights the importance of auditing existing risk score predictors for information content across groups, and demonstrates that obtaining informative calibrated predictions can improve fair selection rules, even when the fairness desiderata are based on parity.

Organization. The manuscript is structured as follows: Section 2 establishes notation and covers the necessary preliminaries; Section 3 provides the technical framework for measuring information in predictors; Section 4 demonstrates how improving information content improves the resulting fair selection rules; and Section 5 describes the merge algorithm for combining and refining multiple predictors. We conclude with a brief discussion of the context of this work and some directions for future investigation.

\subsection{Related Works}

The influential work of [11] provided two observations that are of particular relevance to the present work. First, [11] emphasized the pitfalls of hoping to achieve "fairness through blindness" by censoring sensitive information during prediction. Second, the work highlighted how enforcing broad-strokes demographic parity conditions - even if desired or expected in fair outcomes - is insufficient to imply fairness. Our results can be viewed as providing further evidence for these perspectives. As discussed earlier, understanding the ways in which fair treatment can fail to provide fair outcomes [8,27] provided much of the motivation for this work. The present work can also be viewed as further investigating the tradeoffs between calibration and parity. Inspired by investigative reporting on the "biases" of the COMPAS recidivism prediction system [1], the incompatability of calibration and parity-based notions of fairness has received lots of attention in recent years [7, 26, 29]. Counterintuitively, our work shows how to leverage calibrated predictors to improve the disparity of the eventual decisions. For a comprehensive overview of the literature on the growing list of approaches to fairness in prediction systems, we recommend the recent encyclopedic survey of [28]. 
A few recent works have (implicitly or explicitly) touched on the relationship between information and fairness. [6] argues that discrimination may arise in prediction systems due to disparity in predictive power; they advocate for addressing discrimination through data collection. Arguably, much of the work on fairness in online prediction [21] can be seen as a way to gather information while maintaining fairness. Recently, issues of information and fairness were also studied in unsupervised learning tasks [30]. From the computational economics literature, [25] presents a simple planning model that draws similar qualitative conclusions to this work, demonstrating the significance of trustworthy information as a key factor in algorithmic fairness. Other works [20,22] have investigated the role of hiding information through strategic signaling. In such settings, it may be strategic for a group to hide information about individuals in order to increase the overall selection rate for the group.

Outside the literature on fair prediction, our notions of information content and refinements are related to other notions from the fields of online forecasting and information theory. In particular, the idea of refinements was first introduced in [10]. The concept of information content of calibrated predictions is related to ideas from the forecasting literature $[15,16]$, including sharpness and proper scoring rules [4]. The concept of a refinement of a calibrated predictor can be seen as a special case of Blackwell's informativeness criterion [2, 9, 10].

\section{PRELIMINARIES}

Basic notation. Let $\mathcal{X}$ denote the domain of individuals and $\mathcal{Y}=\{0,1\}$ denote the binary outcome space. We assume that individuals and their outcomes are jointly distributed according to $\mathcal{D}$ supported on $\mathcal{X} \times \mathcal{Y}$. Let $x, y \sim \mathcal{D}$ denote an independent random draw from $\mathcal{D}$. For a subpopulation $S \subseteq \mathcal{X}$, we use the shorthand $x, y \sim \mathcal{D}_{S}$ to be the data distribution conditioned on $x \in S$, and $x \sim S$ to denote a random sample from the marginal distribution over $\mathcal{X}$ conditioned on membership in $S$.

Predictors. A basic goal in learning is to find a classifier $f: \mathcal{X} \rightarrow\{0,1\}$ that given $x \sim \mathcal{X}$ drawn from the marginal distribution over individuals, accurately predicts their outcome $y$. One common strategy for binary classification first maps individuals to a real-valued score using a predictor $z: \mathcal{X} \rightarrow[0,1]$ and then selects individuals on the basis of this score. We denote by $\operatorname{supp}(z)$ the support of $z$. We denote by $p^{*}: \mathcal{X} \rightarrow[0,1]$ the Bayes optimal predictor, where $p^{*}(x)=\operatorname{Pr}_{\mathcal{D}}[y=1 \mid x]$ represents the inherent uncertainty in the outcome given the individual. Equivalently, for each individual $x \in \mathcal{X}$, their outcome $y$ is drawn independently from $\operatorname{Ber}\left(p^{*}(x)\right)$, the Bernoulli distribution with expectation $p^{*}(x)$. While we use $[0,1]$ to denote the codomain of predictors, throughout this work, we assume that the set of individuals is finite and hence, the support of any predictor is a discrete, finite subset of the interval.

Risk score distributions. Note that there is a natural bijection between predictors and score distributions. A predictor $z$, paired with the marginal distribution over $\mathcal{X}$, induces a score distribution, which we denote $\mathcal{R}^{z}$, supported on $[0,1]$, where the probability density function is given as $\mathcal{R}^{z}(v)=\operatorname{Pr}_{x \sim \mathcal{X}}[z(x)=v]$. For a subpopulation $S \subseteq \mathcal{X}$, we denote by $\mathcal{R}_{S}^{z}$ the score distribution conditioned on $x \in S$.

Calibration. A useful property of predictors is called calibration, which implies that the scores can be interpreted meaningfully as the probability that an individual will result in a positive outcome. Calibration has been studied extensively in varied contexts, notably in forecasting and online prediction (e.g. [13]), and recently as a fairness desideratum [8, 18, 26, 29]; the definition we use is adapted from the fairness literature.

Definition (Calibration). A predictor $z: \mathcal{X} \rightarrow[0,1]$ is calibrated on a subpopulation $S \subseteq \mathcal{X}$ if for all $v \in \operatorname{supp}(z), \operatorname{Pr}_{x, y \sim \mathcal{D}_{S}}[y=1 \mid z(x)=v]=v$. 
For convenience, we use the notation $S_{z(x)=v}=\{x \in S: z(x)=v\}$. Note that we can equivalently define calibration with respect to the Bayes optimal predictor, where $z$ is calibrated on $S$ if for all $v \in \operatorname{supp}(z), \mathbf{E}_{x \sim S_{z(x)=v}}\left[p^{*}(x)\right]=v$. Operationally in proofs, we end up using this definition of calibration. This formulation also makes clear that $p^{*}$ is calibrated on every subpopulation.

Parity-based fairness. As a notion of fairness, calibration aims to ensure similarity between predictions and the true outcome distribution. Other fairness desiderata concern disparity in prediction between subpopulations on the basis of a sensitive attribute. For simplicity, we will imagine individuals are partitioned into two subpopulations $A, B \subseteq \mathcal{X}$; we will overload notation and use $\{A, B\}$ to denote the names of the attributes as well. We let $\mathcal{A}: \mathcal{X} \rightarrow\{A, B\}$ map individuals to their associated attribute. The most basic notion of parity is demographic parity (also sometimes called statistical parity in the literature), which states that the selection rate of individuals should be independent of the sensitive attribute.

Definition (Demographic Parity [11]). A selection rule $f: \mathcal{X} \rightarrow\{0,1\}$ satisfies demographic parity if: $\mathbf{P r}_{x \sim X}[f(x)=1 \mid \mathcal{A}(x)=A]=\operatorname{Pr}_{x \sim X}[f(x)=1 \mid \mathcal{A}(x)=B]$.

One critique of demographic parity is that the notion does not take into account the actual qualifications of groups (i.e. no dependence on $y$ ). Another popular parity-based notion, called equalized opportunity, addresses this criticism by enforcing parity of false negative rates across groups.

Definition (EQualized opportunity [17]). A selection rule $f: \mathcal{X} \rightarrow\{0,1\}$ satisfies equalized opportunity if: $\operatorname{Pr}_{x, y \sim \mathcal{D}}[f(x)=0 \mid y=1, \mathcal{A}(x)=A]=$

$$
\operatorname{Pr}_{x, y \sim \mathcal{D}}[f(x)=0 \mid y=1, \mathcal{A}(x)=B] .
$$

In addition to these fairness concepts, the following properties of a selection rule will be useful to track: true positive rate, $\operatorname{TPR}(f)=\operatorname{Pr}_{x, y \sim \mathcal{D}}[f(x)=1 \mid y=1] ;$ false positive rate, $\operatorname{FPR}(f)=$ $\operatorname{Pr}_{x, y \sim \mathcal{D}}[f(x)=1 \mid y=0]$; and positive predictive value, $\operatorname{PPV}(f)=\mathbf{P r}_{x, y \sim \mathcal{D}}[y=1 \mid f(x)=1]$.

\section{MEASURING INFORMATION IN BINARY PREDICTION}

In this section, we give a self-contained exposition of a formal notion of information content in calibrated predictors. These notions have been studied extensively in the forecasting literature (see $[15,16]$ and references therein), but are less common in the literature on computational and statistical learning theory. Our notion of information content can be derived from the Brier scoring rule [4]. We defer proofs of this section to [14].

In the context of binary prediction, a natural way to measure the "informativeness" of a predictor is by the uncertainty in an individual's outcome given their score. We quantify this uncertainty using variance. ${ }^{1}$ For $p \in[0,1]$, the variance of a Bernoulli random variable with expected value $p$ is given as $\operatorname{Var}(\operatorname{Ber}(p))=p \cdot(1-p)$. Note that variance is a strictly concave function in $p$ and is maximized at $p=1 / 2$ and minimized $p \in\{0,1\}$; that is, a Bernoulli trial with $p=1 / 2$ is maximally uncertain whereas a trial with $p=0$ or $p=1$ is perfectly certain. Consider a random draw $x, y \sim \mathcal{D}$ If $z$ is a calibrated predictor, then given $x$ and $z(x)$, the conditional distribution over $y$ follows a Bernoulli distribution with expectation $z(x)$. This observation suggests the following defintion.

Definition (Information content). Suppose for $S \subseteq \mathcal{X}, z: \mathcal{X} \rightarrow[0,1]$ is calibrated on $S$. The information content of $z$ on $S$ is given as $I_{S}(z)=1-4 \cdot \mathbf{E}_{x \sim S}[z(x)(1-z(x))]$.

\footnotetext{
${ }^{1}$ Alternatively, we could measure uncertainty through Shannon entropy (in fact, any function that admits a Bregman divergence). The generality of the approach is made clear in [16]. In [14], we show that notions of information that arise from Shannon entropy are effectively interchangeable with those that arise from variance. We elect to work with variance primarily because it simplifies the analysis in Section 5.
} 
For a calibrated $z$, we use $I(z)=I_{X}(z)$ to denote the "information content of $z$ ". The factor 4 in the definition of information content acts as a normalization factor such that $I(z) \in[0,1]$. At the extremes, a perfectly informative predictor has information content 1 , whereas a calibrated predictor that always outputs $1 / 2$ has 0 information.

This formulation of information content as uncertainty in a binary outcome is intuitive in the context of binary classification. In some settings, however, it may be more instructive to reason about risk score distributions directly. A conceptually different approach to measuring informativeness of a risk score distribution might track the uncertainty in the true (Bayes optimal) risk, given the predicted risk score.

Consider a random variable $P_{z(x)=v}^{*}$ that takes value $p^{*}(x)$ for $x$ sampled from the individuals with score $z(x)=v$; that is, $P_{z(x)=v}^{*}$ equals the true risk for an individual sampled amongst those receiving predicted risk score $v$. We can measure the uncertainty in this random variable by tracking its variance given $z(x)=v$; the higher the variance, the less information the risk score distribution $\mathcal{R}_{z}$ provides about the true risk score distribution $\mathcal{R}_{p^{*}}$. Again, let $S_{z(x)=v}=\{x \in S: z(x)=v\}$ Consider the variance in $P_{v}^{*}$ given as $\operatorname{Var}\left[P_{z(x)=v}^{*}\right]=\mathbf{E}_{x \sim S_{z(x)=v}}\left[\left(p^{*}(x)-v\right)^{2}\right]$. We define the information loss by taking an expectation of this conditional variance over the score distribution induced by $z$.

Definition (Information loss). For $S \subseteq \mathcal{X}$, suppose a predictor $z: \mathcal{X} \rightarrow[0,1]$ is calibrated on $S$. The information loss of $z$ on $S$ is given as:

$L_{S}\left(p^{*} ; z\right)=4 \cdot \mathbf{E}_{v \sim \mathcal{R}_{S}^{z}} \mathbf{E}_{x \sim S_{z(x)=v}}\left[\left(p^{*}(x)-v\right)^{2}\right]$.

Again, the factor 4 is simply to normalize the information loss into the range $L\left(p^{*} ; z\right) \in[0,1]$. This loss is maximized when $p^{*}$ is a $50: 50$ mix of $\{0,1\}$ but $z$ always predicts $1 / 2$; the information loss is minimized for $z=p^{*}$. We observe that this notion of information loss is actually proportional to the expected squared error of $z$ with respect to $p^{*}$; that is, $\mathbf{E}_{v \sim \mathcal{R}_{S}^{z}} \mathbf{E}_{x \sim S_{z(x)=v}}\left[\left(p^{*}(x)-v\right)^{2}\right]=$ $\mathbf{E}_{x \sim S}\left[\left(p^{*}(x)-z(x)\right)^{2}\right]$. Thus, for calibrated predictors, we can interpret the familiar squared loss between a predictor and the Bayes optimal predictor as a notion of information loss.

Connecting information content and information loss. As we defined them, information content and information loss seem like conceptually different ways to measure uncertainty in a predictor. Here, we show that they actually capture the same notion. In particular, we can express information loss of $z$ as the difference in information content of $p^{*}$ and that of $z$.

Proposition 3.1. Let $p^{*}: \mathcal{X} \rightarrow[0,1]$ denote the Bayes optimal predictor. Suppose for $S \subseteq \mathcal{X}$, $z: \mathcal{X} \rightarrow[0,1]$ is calibrated on $S$. Then, $L_{S}\left(p^{*} ; z\right)=I_{S}\left(p^{*}\right)-I_{S}(z)$.

Because the information loss is a nonnegative quantity, Proposition 3.1 also formalizes the inutition that the Bayes optimal predictor is the most informative predictor; for $z \neq p^{*}, I(z)<$ $I\left(p^{*}\right) \leq 1$. Ideally, in order to evaluate the information disparity across groups, we would compare the information lost from $p^{*}$ to $z$ across $A$ and $B$. But because the definition of information loss depends on the true score distribution $p^{*}$, in general, it's impossible to directly compare the loss. Still, if we believe that $p^{*}(x)$ is similarly distributed across $A$ and $B$, then the information contents $I_{A}(z)$ and $I_{B}(z)$ of the observed risk scores allow us to directly compare the loss.

\subsection{Incorporating information via refinements}

We have motivated the study of informativeness in prediction with the intuition that as information content improves, so too will the resulting fairness and utility of the decisions derived from the predictor. Without further assumptions, however, this line of reasoning turns out to be overlyoptimistic. For instance, consider a setting where the expected utility of lending to individuals is 
positive if $z(x)>\tau$ for some fixed threshold $\tau$ (and negative otherwise). In this case, information about individuals whose $p^{*}(x)$ is significantly below $\tau$ is not especially useful. Information content $I(z)$ is a global property of $z$, whereas the quantities that affect the utility and fairness directly, like PPV or TPR are conditional properties. Even if $I\left(z^{\prime}\right)>I(z)$, it could be that $z^{\prime}$ has lost information about an important subpopulation compared to $z$, compensating with lots of information about the unqualified individuals. Still, we would like to characterize ways in which more information is definitively "better." Intuitively, more information is better when we don't have to give up on the information in the current predictor, but rather refine the information contained in the predictions further. The following definition, equivalent to a notion proposed in [10], formalizes this idea.

Definition (Refinement). For $S \subseteq \mathcal{X}$, suppose $z, z^{\prime}: \mathcal{X} \rightarrow[0,1]$ are calibrated on $S$. $z^{\prime}$ is a refinement of $z$ on $S$ if for all $v \in \operatorname{supp}(z), \mathbf{E}_{x \sim S_{z(x)=v}}\left[z^{\prime}(x)\right]=v$.

That is, we say that $z^{\prime}$ refines $z$ if $z^{\prime}$ maintains the same expectation over the level sets $S_{z(x)=v}$. To understand why this property makes sense in the context of maintaining information from $z$ to $z^{\prime}$, suppose the property was violated: that is, there is some $v \in \operatorname{supp}(z)$ such that $\mathbf{E}_{x \sim S_{z(x)=v}}\left[z^{\prime}(x)\right] \neq v$. This disagreement provides evidence that $z$ has some consistency with the true risk that $z^{\prime}$ is lacking; because $z$ is calibrated, $\mathbf{E}_{x \sim S_{z(x)=v}}[z(x)]=v=\mathbf{E}_{x \sim S_{z(x)=v}}\left[p^{*}(x)\right]$. In other words, even if $z^{\prime}$ has greater information content, it may not be consistent with the content of $z$.

Another useful perspective on refinements is through measuring the information on each of the sets $S_{z(x)=v}$. Restricted to $S_{z(x)=v}, z$ has minimal information content - its predictions are constant - whereas $z^{\prime}$ may vary. Because $z^{\prime}$ is calibrated and maintains the expectation over $S_{z(x)=v}$, we can conclude that $I_{S_{z(x)=v}}(z) \leq I_{S_{z(x)=v}}\left(z^{\prime}\right)$ for each of the partitions. This perspective highlights the importance of requiring calibration in the definition of refinements. Indeed, because a refinement is a calibrated predictor, refinements cannot make arbitrary distinctions in predictions, so any additional distinctions on the level sets of the original predictor must represent true variability in $p^{*}$. We draw attention to the similarity between the definition of a refinement and the definition of calibration. Note that $p^{*}$ is a refinement of every calibrated predictor. Indeed, one way to interpret a refinement is as a "candidate" Bayes optimal predictor. Carrying this intuition through, we note that the only property of $p^{*}$ we used in the proof of Proposition 3.1 is that it is a refinement of a calibrated $z$. Thus, we can immediately restate the proposition in terms of refinements.

Proposition 3.2. Suppose for $S \subseteq \mathcal{X}, z, z^{\prime}: \mathcal{X} \rightarrow[0,1]$ are calibrated on $S$. If $z^{\prime}$ refines $z$ on $S$, then $L_{S}\left(z^{\prime} ; z\right)=I_{S}\left(z^{\prime}\right)-I_{S}(z)$.

This characterization further illustrates the notion that a refinement $z^{\prime}$ could plausibly be the true risk given the information in the current predictions $z$. In particular, because $L_{S}\left(z^{\prime} ; z\right)>0$, we get $I_{S}\left(z^{\prime}\right)>I_{S}(z)$ for any refinement $z^{\prime} \neq z$.

In the context of fair prediction, we want to ensure that the information content on specific protected subpopulations does not decrease. Indeed, in this case, it may be important to ensure that the predictions are refined, not just overall, but also on the sensitive subpopulations. Indeed, it is possible to construct examples where $z, z^{\prime}: \mathcal{X} \rightarrow[0,1]$ that are each calibrated on two subpopulations $A, B \subseteq \mathcal{X}, z^{\prime}$ refines $z$ on $\mathcal{X}$ overall, but $z^{\prime}$ loses information about the subpopulation $A$. (See [14].) Such negative examples highlight the importance of incorporating all the information available (e.g. group membership), not only at the time of decision-making, but also along the way when developing predictors; it serves as yet another rebuke of the approach of "fairness through blindness" [11]. 


\section{THE VALUE OF INFORMATION IN FAIR PREDICTION}

In this section, we argue that reasoning about the information content of calibrated predictors provides a lens into understanding how to improve the utility and fairness of predictors, even when the eventual fairness desideratum is based on parity. We discuss a prediction setting based on that of [27] where a lender selects individuals to give loans from a pool of applicants. While we use the language of predicting creditworthiness, the setup is generic and can be applied to diverse prediction tasks. [27] introduced a notion of "delayed impact" of selection policies, which models the potential negative impact on communities of enforcing parity-based fairness as a constraint. We revisit the question of delayed impact as part of a broader investigation of the role of information in fair prediction. We begin with an overview of the prediction setup. Then, we prove our main result: refining the underlying predictions used to choose a selection policy results in an improvement in utility, parity, or impact (or a combination of the three).

\subsection{Fair prediction setup}

When deciding how to select qualified individuals, the lender's goal is to maximize some expected utility. Specifically, the utility function $u:[0,1] \rightarrow[-1,1]$ specifies the lender's expected utility from an individual based on their score and a fixed threshold $\tau_{u} \in[0,1]$ as given as $u(p)=p-\tau_{u}$. When considering delayed impact, we will measure the expected impact per subpopulation. The impact function $\ell:[0,1] \rightarrow[-1,1]$ specifies the expected benefit to an individual from receiving a loan based on their score and a fixed threshold $\tau_{\ell} \in[0,1]$ given as $\ell(p)=p-\tau_{\ell}$. [27] models riskaversion of the lender by assuming that $\tau_{u}>\tau_{\ell}$; that is, by choosing accepting individuals with $z(x) \in\left(\tau_{\ell}, \tau_{u}\right)$, the impact on subpopulations may improve beyond the lender's utility-maximizing policy.

In this setup, we allow the lender to pick a (randomized) group-sensitive selection policy $f$ : $[0,1] \times\{A, B\} \rightarrow[0,1]$ that selects individuals on the basis of a predicted score and their sensitive attribute. That is, the selection policy makes decisions about individuals via their score according to some calibrated predictor $z: \mathcal{X} \rightarrow[0,1]$ and their sensitive attribute $\mathcal{A}: \mathcal{X} \rightarrow\{A, B\}$; for every individual $x \in \mathcal{X}$, the probability that $x$ is selected is given as $f(z(x), \mathcal{A}(x))$.

We will restrict our attention to threshold policies; that is, for sensitive attribute $A$ (resp., $B$ ), there is some $\tau_{A} \in[0,1]$, such that $f(v, A)$ is given as $f(v, A)=1$ if $v>\tau_{A}, f(v, A)=0$ if $v<\tau_{A}$ and $f(v, A)=p_{A}$ for $v=\tau_{A}$, where $p_{A} \in[0,1]$ is a probability used to randomly break ties on the threshold. The motivation for focusing on threshold policies is their intuitiveness, widespread use, computational efficiency ${ }^{3}$. The restriction to threshold policies is justified formally in [27] by the fact that the optimal decision rule in our setting can be specified as a threshold policy under both demographic parity and equalized opportunity.

Given this setup, we can write the expected utility $U^{z}(f)$ of a policy $f$ based on a calibrated predictor $z$, that is calibrated on both subpopulations, $A$ and $B$, as follows.

$$
U^{z}(f)=\sum_{S \in\{A, B\}} \operatorname{Pr}_{x \sim X}[x \in S] \cdot\left(\sum_{v \in \operatorname{supp}(z)} \mathcal{R}_{S}^{z}(v) \cdot f(v, S) \cdot u(v)\right)
$$

Recall, $\mathcal{R}_{S}^{z}(v)=\operatorname{Pr}_{x \sim S}[z(x)=v]$.

\footnotetext{
${ }^{2}$ Assuming such an affine utility function is equivalent to assuming that the lender receives $u_{+}$from repayments, $u_{-}$from defaults, and 0 from individuals that do not receive loans. In this case, the expected utility for score $p$ is $p u_{+}+(1-p) u_{-}=$ $c_{u} \cdot u(p)$ for some constant $c_{u}$

${ }^{3}$ Indeed, without the restriction to threshold policies, many of the information-theoretic arguments become easier at the expense of computational cost. As $z^{\prime}$ is a refinement of $z$, we can always simulate decisions derived from $z$ given $z^{\prime}$, but in general, we cannot do this efficiently.
} 
Similarly, the expected impact over the subpopulations $S \in\{A, B\}$ are given as

$$
\operatorname{Imp}_{S}^{z}(f)=\sum_{v \in \operatorname{supp}(z)} \mathcal{R}_{S}^{z}(v) \cdot f(v, S) \cdot \ell(v)
$$

Often, it may make sense to constrain the net impact to each group as defined in (2) to be positive, ensuring that the selection policies do not do harm as in [27].

The following quantities will be of interest to the lender when choosing a selection policy $f$ as a function of $z$. First, the lender's overall utility $U(f)$ is given as in (1). In the name of fairness, the lender may also be concerned about the disparity of a number of quantities. We will show below that these quantities can be written as a linear function of the selection rule. In particular, for $S \in\{A, B\}$ demographic parity, which serve as our running example, compares the selection rates $\beta_{S}^{z}(f)=\operatorname{Pr}_{x \sim S}[x$ selected by $f]=\sum_{v \in \operatorname{supp}(z)} \mathcal{R}_{S}^{z}(v) \cdot f(v, S)$.

We may also be concerned about comparing the true positive rates, $\operatorname{TPR}_{S}^{z}(f)=\operatorname{Pr}[x$ selected by $f \mid y=$ $1]=\frac{1}{r_{S}} \cdot \sum_{v \in \operatorname{supp}(z)} \mathcal{R}_{S}^{z}(v) \cdot f(v, S) \cdot v$ and false positive rates, $\operatorname{FPR}_{S}^{z}(f)=\operatorname{Pr}[x$ selected by $f \mid y=$ $0]=\frac{1}{1-r_{S}} \cdot \sum_{v \in \operatorname{supp}(z)} \mathcal{R}_{S}^{z}(v) \cdot f(v, S) \cdot(1-v)$, where $r_{S}$ represents the base rate of the subpopulation $S$; that is, $r_{S}=\operatorname{Pr}_{(x, y) \sim D_{S}}[y=1]$. Another quantity we will track is the positive predictive value, $\operatorname{PPV}_{S}^{z}(f)=\operatorname{Pr}[y=1 \mid x$ selected by $f]=\frac{1}{\beta_{S}^{z}(f)} \cdot\left(\sum_{v \in \operatorname{supp}(z)} \mathcal{R}_{S}^{z}(v) \cdot f(v, S) \cdot v\right)$. Note that $\operatorname{PPV}_{S}^{z}(f)$ is not a linear function of $f(v, S)$ values, but as we never use positive predictive values directly in the optimizations for choosing a selection policy (or in a parity-based fairness definition), the optimizations are still a linear program. For notational convenience, we may drop the superscript of these quantities when $z$ is clear from the context.

\subsection{Refinements in the service of fair prediction}

Note that all of the quantities described in Section 4.1 can be written as linear functions of $f(v, S)$. Given a fixed predictor $z: \mathcal{X} \rightarrow[0,1]$, we can expand the quantities of interest; in particular, we note that the linear functions over $f(v, S)$ can be rewritten as linear functions over $z(x)$, where the quantities depend on $x$ only through the predictor $z$. In this section, we show how refining the predictor used for determining the selection rule can improve the utility, parity, and impact of the optimal selection rule. By the observations above, we can formulate a generic policy-selection problem as a linear program where $z$ controls many coefficients in the program. When we refine $z$, we show that the value of the program increases. Recalling that different contexts may call for different notions of fairness, we consider a number of different linear programs the lender (or regulator) might choose to optimize. At a high-level, the lender can choose to maximize utility, minimize disparity, or maximize positive impact on groups, while also maintaining some guarantees over the other quantities.

We will consider selection policies given a fixed predictor $z: \mathcal{X} \rightarrow[0,1]$. Note that the paritybased fairness desiderata we consider are of the form $h_{A}^{z}(f)=h_{B}^{z}(f)$ for some $h \in\{\beta, \mathrm{TPR}, \mathrm{FPR}\}$; rather than requiring equality, we will consider the disparity $\left|h_{A}^{z}(f)-h_{B}^{z}(f)\right|$ and in some cases, constrain it to be less than some constant $\varepsilon$. We also use $t_{i}, t_{u}$ to denote lower bounds on the desired impact and utility, respectively. For simplicity's sake, we assume that $B$ is the "protected" group, so we only enforce the positive impact constraint for this group; more generally, we could include an impact constraint for each group. Formally, we consider the following constrained optimizations. 


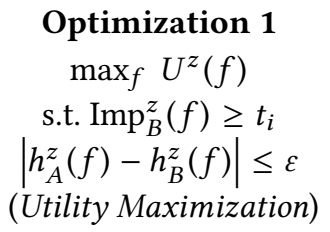

\author{
Optimization 3 \\ $\max _{f} \operatorname{Imp}_{B}^{z}(f)$ \\ s.t. $U^{z}(f) \geq t_{u}$ \\ $\left|h_{A}^{z}(f)-h_{B}^{z}(f)\right| \leq \epsilon$ \\ (Impact Maximization)
}

LEMma 4.1. Let $h \in\{\beta, \mathrm{TPR}, \mathrm{FPR}\}$. Given a calibrated predictor $z: \mathcal{X} \rightarrow[0,1]$, Optimization 1,2, and 3 are linear programs in the variables $f(v, S)$ for $v \in \operatorname{supp}(z)$ and $S \in\{A, B\}$. For each program, there is an optimal solution $f^{*}$ that is a threshold policy.

For the proof of the lemma, see [14].

Improving the cost of fairness. We argue that in all of these optimizations, increasing information through refinements of the current predictor on both the subpopulations $A$ and $B$ improves this value of the program. We emphasize that this conclusion is true for all of the notions of parity-based fairness we mentioned above. Thus, independent of the exact formulation of fair selection that policy-makers deem appropriate, information content is a key factor in determining the properties of the resulting selection rule. We formalize this statement in the following theorem.

Theorem 4.2. Let $z, z^{\prime}: \mathcal{X} \rightarrow[0,1]$ be two predictors that are calibrated on disjoint subpopulations $A, B \subseteq \mathcal{X}$. For any of the Optimization 1, 2, 3 and their corresponding fixed parameters, let OPT $(z)$ denote their optimal value under predictor $z$. If $z^{\prime}$ refines $z$ on $A$ and $B$, then $O P T\left(z^{\prime}\right) \geq O P T(z)$ for Optimization 1, 3 and $\mathrm{OPT}\left(z^{\prime}\right) \leq \mathrm{OPT}(z)$ for Optimization 2.

One way to understand Theorem 4.2 is through a "cost of fairness" analysis. Focusing on the utility maximization setting, let $U^{*}$ be the maximum unconstrained utility achievable by the lender given the optimal predictions $p^{*}$. Let $\operatorname{OPT}(z)$ be the optimal value of Optimization 1 using predictions $z$; that is, the best utility a lender can achieve under a parity-based fairness constraint $(\varepsilon=0)$ and positive impact constraint $\left(t_{i}=0\right)$. If we take the cost of fairness to be the difference between these optimal utilities, $U^{*}-\mathrm{OPT}(z)$, then Theorem 4.2 says that by refining $z$ to $z^{\prime}$, the cost of fairness decreases with increasing informativeness; that is, $U^{*}-\operatorname{OPT}(z) \geq U^{*}-\operatorname{OPT}\left(z^{\prime}\right)$. This corollary of Theorem 4.2 corroborates the idea that in some cases the high perceived cost associated with requiring fairness might actually be due to the low informativeness of the predictions in minority populations. No matter what the true $p^{*}$ is, this cost will decrease as we increase information content by refining subpopulations.

For $S \in\{A, B\}$, we use $\operatorname{TPR}_{S}^{z}(\beta)$ to denote the true positive rate of the threshold policy with selection rate $\beta$ for the subpopulation $S$ while using the predictor $z^{4}$. Similarly, $\operatorname{PPV}_{S}^{z}(\beta), \operatorname{FPR}_{S}^{z}(\beta)$ are defined. The following lemma, which plays a key role in each proof, shows that refinements broadly improve selection policies across these three statistics of interest.

Lemma 4.3. If $z^{\prime}$ is a refinement of $z$ on subpopulations $A$ and $B$, then for $S \in\{A, B\}$, for all $\beta \in[0,1]$,

$$
\operatorname{TPR}_{S}^{z^{\prime}}(\beta) \geq \operatorname{TPR}_{S}^{z}(\beta), \quad \operatorname{FPR}_{S}^{z^{\prime}}(\beta) \leq \operatorname{FPR}_{S}^{z}(\beta), \quad \operatorname{PPV}_{S}^{z^{\prime}}(\beta) \geq \operatorname{PPV}_{S}^{z}(\beta) .
$$

Proof of Lemma 4.3. Note that for a fixed selection rate $\beta$, PPV is maximized by picking the top-most $\beta$ fraction of the individuals ranked according to $p^{*}$, i.e. a threshold policy that selects a $\beta$-fraction of the individuals using the Bayes optimal predictor $p^{*}$. Similarly, for a fixed selection rate, the TPR and FPR values are also optimized under a threshold selection policy that uses the Bayes optimal predictor $p^{*}$.

Recall, we can interpret a refinement as a "candidate" optimal predictor. In particular, because $z^{\prime}$ refines $z$ over $A$ and $B$, we know that $z$ is calibrated not only with respect to the true optimal

\footnotetext{
${ }^{4}$ Given a predictor, there is a bijection between selection rates and threshold policies.
} 
predictor $p^{*}$, but also with respect to the refinement $z^{\prime}$ on both subpopulations. Imagining a world in which $z^{\prime}$ is the Bayes optimal predictor, the PPV, TPR, and FPR must be no worse under a threshold policy derived from $z^{\prime}$ compared to that of $z$ by the initial observation. Thus, the lemma follows. $\square$

Using Lemma 4.3, we prove Theorem 4.2. For the complete proof, see [14]. The outline of the proof is as follows. Let $f$ be any threshold selection policy under the predictor $z$. Using $f$, we will construct a selection policy $f^{\prime}$ that uses the refined score distribution $z^{\prime}$ such that where $U^{z^{\prime}}\left(f^{\prime}\right) \geq U^{z}(f)$, $\operatorname{Imp}_{B}^{z^{\prime}}\left(f^{\prime}\right) \geq \operatorname{Imp}_{B}^{z}(f)$, and $h_{A}^{z^{\prime}}\left(f^{\prime}\right)=h_{A}^{z}(f)$ and $h_{B}^{z^{\prime}}\left(f^{\prime}\right)=h_{B}^{z}(f)$. Here, $h \in\{\beta$, TPR, FPR $\}$ specifies the parity-based fairness definition being used. Thus, taking $f$ to be the optimal solution to any of the Optimizations 1, 2 or 3, we see that $f^{\prime}$ is a feasible solution to the same optimization and has the same or a better objective value compared to $f$. Therefore, after optimization, objective values can only get better.

In words, we are saying that refined predictors allow us to get better utility and impact as the original predictor while keeping the parity values the same (for e.g., while keeping the selection rates the same in both subpopulations).

\section{A MECHANISM FOR REFINING PREDICTORS}

In this section, we outline a mechanism for obtaining refinements of predictors. We start by describing an algorithm, merge, which given two calibrated predictors, produces a new refined predictor that incorporates the information from both predictors (in a sense we make formal). For notational convenience, we describe how to refine a predictor over $\mathcal{X}$. The arguments here extend easily to refining over a partition of $\mathcal{X}$ by refining each part separately.

The most obvious way to demonstrate that a predictor $z$ can be refined would be to exhibit some calibrated $q: \mathcal{X} \rightarrow[0,1]$ such that $I(q)>I(z)$. That said, expecting that we could obtain such a $q$ seems to sidestep the question of how to update a predictor to improve its information content. Even if we were able to obtain some $q$ where $I(q)>I(z)$, it is not clear that $q$ would be a "better" predictor. In particular, $q$ might contain different information than $z$; recall that such examples motivated the definition of a refinement in the first place. Still, intuitively, if $q$ is not a refinement of $z$ and contains very different information than $z$, then $q$ should be useful in identifying ways to improve the informativeness of $z$. Further, this intuition does not seem to rely on the fact that $I(q)>I(z)$; as long as $q$ contains information that isn't "known" to $z$, then incorporating the information into $z$ should reduce the information loss.

To formalize this line of reasoning, first, we need to make precise what we mean when we say that $q$ is far from refining $z$. Recalling the definition of a refinement, consider the logical negation of the statement that " $q$ refines $z$."

$$
\neg\left(\forall v \in \operatorname{supp}(z): \underset{x \sim X_{z(x)=v}}{\mathbf{E}}[q(x)]=v\right) \Longleftrightarrow \exists v \in \operatorname{supp}(z): \underset{x \sim X_{z(x)=v}}{\mathbf{E}}[q(x)] \neq v .
$$

Extending this logical formulation, we define the following divergence to capture quantitatively how far $q$ is from refining $z$.

Definition (Refinement Distance). Let $q, z: \mathcal{X} \rightarrow[0,1]$ be calibrated predictors. The refinement distance from $z$ to $q$ is given as:

$D_{R}(z ; q)=\sum_{v \in \operatorname{supp}(z)} \mathcal{R}^{z}(v) \cdot\left|\mathbf{E}_{x \sim X_{z(x)=v}}[q(x)]-v\right|$.

Note that $D_{R}(z ; q)$ is not symmetric; in particular, if $q$ refines $z$ and contains more information $I(q)>I(z)$, then $D_{R}(z ; q)=0$, but $D_{R}(q ; z)>0$. Intuitively, the refinement distance averages the refinement "disagreements" over all values in the support. We show that, under calibration, these disagreements can be reconciled to improve the overall information content. With the notion of 
refinement distance in place, we can state the main algorithmic result - a simple algorithm for aggregating the information of multiple calibrated predictors into a single calibrated predictor.

Theorem 5.1. Given two calibrated predictors $q, z: \mathcal{X} \rightarrow[0,1]$, Algorithm 1 produces a new calibrated predictor $\rho: \mathcal{X} \rightarrow[0,1]$ such that $\rho$ is a refinement of both $z$ and $q$. Further, $I(\rho)>$ $\max \left\{I(z)+4 \cdot D_{R}(q ; z)^{2}, I(q)+4 \cdot D_{R}(z ; q)^{2}\right\}$.

We state the theorem generally, making no assumptions about $D_{R}(q ; z)$ or $D_{R}(z ; q)$. In particular, as we alluded to earlier, if $q$ already refines $z$, then $D_{R}(z ; q)=0$, so there will be no information gain. Algorithm 1, which we refer to as merge, describes the procedure. We describe the algorithm in the statistical query model, where we assume query access to aggregate statistics about $p^{*}(x)$. The merge algorithm builds a new calibrated predictor $\rho$ from $q$ and $z$ by considering the set of individuals who receive $q(x)=u$ and $z(x)=v$ for each $u \in \operatorname{supp}(q)$ and $v \in \operatorname{supp}(z)$. For each of these sets, the merged predictor adjusts the prediction to have the correct expectation. The proof of Theorem 5.1 follows from a standard potential function analysis; further, the sample complexity needed to answer the statistical queries accurately is bounded. These proofs are included in [14].

Algorithm 1: merge $(z, q)$

Given: $z, q: \mathcal{X} \rightarrow[0,1]$ calibrated predictors

Output: $\rho: \mathcal{X} \rightarrow[0,1]$ a refinement of $z$ and $q$

- Let $\mathcal{Z}=\left\{X_{z(x)=v}: v \in \operatorname{supp}(z)\right\}$

- Let $Q=\left\{X_{q(x)=u}: u \in \operatorname{supp}(q)\right\}$

- For $Z_{v} \in \mathcal{Z}$ and $Q_{u} \in Q$ :

$-\mathcal{X}_{v u}=Z_{v} \cap Q_{u}$

$-\rho(x) \leftarrow \underset{x \sim X_{v u}}{\mathbf{E}}\left[p^{*}(x)\right]$

Interpreting the updates. As described, the merge algorithm takes two different calibrated predictors and combines them into a refinement. In settings where the lender wants to combine predictions from different sources, this algorithmic model is naturally well-motivated. Still, there are other settings where the merge algorithm can be applied. One natural way we can specify new information content is by giving the predictor an additional feature. Specifically, consider some a boolean feature $\phi: \mathcal{X} \rightarrow\{0,1\}$. We define the predictor $q_{\phi}: \mathcal{X} \rightarrow[0,1]$ to be $q_{\phi}(x)=$ $\mathbf{E}_{x^{\prime} \sim \mathcal{X}}\left[p^{*}\left(x^{\prime}\right) \mid \phi\left(x^{\prime}\right)=\phi(x)\right]$. This predictor gives the expected value over the set of individuals where $\phi(x)=1$ (resp., $\phi(x)=0$ ); thus, the predictor is calibrated. Merging $z$ with $q_{\phi}$ incorporates the information in the boolean feature $\phi$ into the predictions of $z$.

\section{DISCUSSION}

The information content of a predictor $z$ can be significantly larger on the majority population $S$ than the minority $T$ for a number of reasons.

- Despite optimal predictions, the individuals in $S$ are inherently more predictable than those in $T$; i.e. $z \approx p^{*}$ and $I_{S}\left(p^{*}\right)>I_{T}\left(p^{*}\right)$. If this (controversial) hypothesis is true, there may be no way to improve the predictions further, and some degree of disparity may be unavoidable. Note that in general this condition cannot be verified from data. Still, the assumption that $I(z)=I\left(p^{*}\right)$ can be falsified by finding a way to give more informative predictions.

- Nontrivial information loss has occurred in $z$ on $T$ compared to $S$; i.e. $L_{S}\left(p^{*} ; z\right)<L_{T}\left(p^{*} ; z\right)$. Such information loss could result from purely information theoretic issues (features used for prediction are not sufficiently expressive in $T$ ), a mix of informational and computational 
issues (not enough data from the minority to learn a predictor from a sufficiently rich hypothesis class), or purely computational issues (suboptimal learning in $T$ due to optimization for $S \cup T$ ). Each source of information disparity has a different own solution (collecting better features, collecting more data, re-training with awareness of the population $T$, respectively), but the tools we present for reasoning about information content apply broadly.

As such, improving the information content of predictions may require collecting additional features or data. Once collected, the merge procedure provides a relatively inexpensive way of incorporating new information into the predictions while retaining certain "quality" of the selection rule. In practice, when the sensitive group $T$ is known, it may make sense to simply retrain the prediction model with awareness of $T$.

Overlapping subpopulations. Often, as highlighted in [18, 23, 24], the subpopulations in need of protection may be hard to anticipate. These recent works have studied notions of multi-fairness that aim to strengthen notions of group fairness by enforcing statistical constraints not just overall, but on a rich family of subgroups. In particular, [18] introduces a notion called multicalibration, which informally, guarantees calibration across all subpopulations specified by a given set system $C$. We observe that the guarantees of multicalibration can be reinterpreted in the language of refinements: a predictor that is multicalibrated with respect to $C$ is simultaneously a refinement for all $q_{c}: \mathcal{X} \rightarrow[0,1]$ where $q_{c}(x)=\mathbf{E}_{x^{\prime} \sim \mathcal{X}}\left[p^{*}\left(x^{\prime}\right) \mid c\left(x^{\prime}\right)=c(x)\right]$ for every $c \in C$. In this sense, multicalibration may be an effective approach to improving information across subpopulations, when the groups that might be experiencing discrimination are unknown or overlapping. An interesting question is whether some of the analysis in the present work can be applied to understand better the connections between multicalibration and the work of [23] which studies the notion of rich subgroup fairness under demographic parity and equalized opportunity.

Choosing fairness constraints. Understanding precisely the guarantees of the specified fairness constraints is particularly important for interpreting the results of Section 4. In particular, refining predictions is guaranteed to improve the value of the stated program. We emphasize the importance of faithfully translating fairness desiderata into mathematical requirements. For instance, suppose policy-makers want to increase representation for historically-disenfranchised populations. An appealing - but misguided - translation of this goal would require demographic parity; intuitively, if the lender is required to have equalized selection rates across groups, they might increase the selection rate in the minority to match that of the majority. Still, demographic parity only requires parity of selection rates which could also be achieved by reducing the selection in the majority. Further, refinements under demographic parity constraints, might cause the selection rates in the minority to decrease. By increasing information, we might uncover that fewer individuals are actually above the tolerable risk than the previous predictions suggested; as such, fewer individuals might be deemed qualified for a loan.

The framework proposed in Section 4 is compatible with a variety of constraints and objectives, including explicitly lower bounding the group selection rates. Thus, increasing the selection rate in a given population can always be achieved by directly constraining the selection rule. An appealing aspect of the framework is that it allows policy-makers to experiment with constraints and objectives to understand the downstream effects of their policies, given the current set of predictions. For instance, policy-makers can evaluate how lower bounding the selection rate in a group will affect the impact of the policy on this group. Such experimentation with the programs from Section 4 may help to guide future policy decisions.

\section{REFERENCES}

[1] Julia Angwin, Jeff Larson, Surya Mattu, and Lauren Kirchner. 2016. Machine Bias: There's software used across the country to predict future criminals. And it's biased against blacks. ProPublica (2016). 
[2] David Blackwell. 1953. Equivalent comparisons of experiments. The annals of mathematical statistics (1953).

[3] Tolga Bolukbasi, Kai-Wei Chang, James Y Zou, Venkatesh Saligrama, and Adam T Kalai. 2016. Man is to computer programmer as woman is to homemaker? debiasing word embeddings. In Neural Information Processing Systems.

[4] Glenn W Brier. 1950. Verification of forecasts expressed in terms of probability. Monthly Weather Review (1950).

[5] Joy Buolamwini and Timnit Gebru. 2018. Gender shades: Intersectional accuracy disparities in commercial gender classification. In $F A T^{*}$.

[6] Irene Chen, Fredrik D Johansson, and David Sontag. 2018. Why Is My Classifier Discriminatory? Neural Information Processing Systems (2018).

[7] Alexandra Chouldechova. 2017. Fair prediction with disparate impact: A study of bias in recidivism prediction instruments. Big Data (2017).

[8] Sam Corbett-Davies and Sharad Goel. 2018. The measure and mismeasure of fairness: A critical review of fair machine learning. arXiv preprint 1808.00023 (2018).

[9] Jacques Crémer. 1982. A simple proof of Blackwell's âĂIJcomparison of experimentsâĂİ theorem. Fournal of Economic Theory (1982)

[10] Morris H DeGroot and Stephen E Fienberg. 1981. Assessing Probability Assessors: Calibration and Refinement. Technical Report. CARNEGIE-MELLON UNIV PITTSBURGH PA DEPT OF STATISTICS.

[11] Cynthia Dwork, Moritz Hardt, Toniann Pitassi, Omer Reingold, and Richard S. Zemel. 2012. Fairness through awareness. In ITCS.

[12] Irfan Faizullabhoy and Aleksandra Korolova. 2018. Facebook's Advertising Platform: New Attack Vectors and the Need for Interventions. arXiv preprint 1803.10099 (2018).

[13] Dean P. Foster and Rakesh V. Vohra. 1998. Asymptotic Calibration. Biometrika (1998).

[14] Sumegha Garg, Michael P Kim, and Omer Reingold. 2019. Tracking and Improving Information in the Service of Fairness. arXiv preprint arXiv:1904.09942 (2019).

[15] Tilmann Gneiting, Fadoua Balabdaoui, and Adrian E Raftery. 2007. Probabilistic forecasts, calibration and sharpness. fournal of the Royal Statistical Society: Series B (Statistical Methodology) (2007).

[16] Tilmann Gneiting and Adrian E Raftery. 2007. Strictly proper scoring rules, prediction, and estimation. F. Amer. Statist. Assoc. (2007).

[17] Moritz Hardt, Eric Price, and Nathan Srebro. 2016. Equality of opportunity in supervised learning. In Neural Information Processing Systems.

[18] Úrsula Hébert-Johnson, Michael P. Kim, Omer Reingold, and Guy N. Rothblum. 2018. Calibration for the (Computationally-Identifiable) Masses. ICML (2018).

[19] Ben Hutchinson and Margaret Mitchell. 2019. 50 Years of Testing (Un)fairness: Lessons for Machine Learning. In $F A T^{*}$.

[20] Nicole Immorlica, Katrina Ligett, and Juba Ziani. 2019. Access to Population-Level Signaling as a Source of Inequality. FAT $^{*}$ (2019).

[21] Matthew Joseph, Michael Kearns, Jamie H. Morgenstern, and Aaron Roth. 2016. Fairness in learning: Classic and contextual bandits. In Neural Information Processing Systems.

[22] Sampath Kannan, Aaron Roth, and Juba Ziani. 2019. Downstream Effects of Affirmative Action. FAT* (2019).

[23] Michael Kearns, Seth Neel, Aaron Roth, and Zhiwei Steven Wu. 2018. Preventing Fairness Gerrymandering: Auditing and Learning for Subgroup Fairness. ICML (2018).

[24] Michael P. Kim, Omer Reingold, and Guy N. Rothblum. 2018. Fairness Through Computationally-Bounded Awareness. Neural Information Processing Systems (2018).

[25] Jon Kleinberg, Jens Ludwig, Sendhil Mullainathan, and Ashesh Rambachan. 2018. Algorithmic Fairness. In AEA Papers and Proceedings.

[26] Jon M. Kleinberg, Sendhil Mullainathan, and Manish Raghavan. 2017. Inherent Trade-Offs in the Fair Determination of Risk Scores. In ITCS.

[27] Lydia T. Liu, Sarah Dean, Esther Rolf, Max Simchowitz, and Moritz Hardt. 2018. Delayed Impact of Fair Machine Learning. In ICML.

[28] Shira Mitchell, Eric Potash, and Solon Barocas. 2018. Prediction-Based Decisions and Fairness: A Catalogue of Choices, Assumptions, and Definitions. arXiv preprint 1811.07867 (2018).

[29] Geoff Pleiss, Manish Raghavan, Felix Wu, Jon M. Kleinberg, and Kilian Q. Weinberger. 2017. On Fairness and Calibration. In Neural Information Processing Systems.

[30] Samira Samadi, Uthaipon Tantipongpipat, Jamie H Morgenstern, Mohit Singh, and Santosh Vempala. 2018. The Price of Fair PCA: One Extra Dimension. In Neural Information Processing Systems.

[31] Andrew D. Selbst, danah boyd, Sorelle A. Friedler, Suresh Venkatasubramanian, and Janet Vertesi. 2019. Fairness and Abstraction in Sociotechnical Systems. In FAT*. 